\title{
Riddle of light: a seminar course for scientists and humanists
}

\section{Boris Stoicheff}

Boris P. Stoicheff, "Riddle of light: a seminar course for scientists and humanists," Proc. SPIE 3831, Sixth International Conference on Education and Training in Optics and Photonics, (16 June 2000); doi: 10.1117/12.388702

Event: Education and Training in Optics and Photonics (ETOP'99), 1999, Cancun, Mexico 


\title{
'The riddle of light': a seminar course for scientists and humanists
}

\author{
Boris P. Stoicheff \\ Department of Physics, University of Toronto, Toronto, ON, Canada M5S 1A7
}

\begin{abstract}
Recently a new program of 'First Year Seminar Courses' was initiated in the Faculty of Arts and Science at the University of Toronto to introduce in-coming undergraduates to the excitement of university scholarship. These are full-year elective courses, and in each course students work closely with a faculty member in groups of no more than 20 students. I shall review my experiences of these past three years in organizing a course on light, optics, and photonics. The primary aim was to use the rich story of light to bring together students of a variety of intellectual interests, in the hope of demonstrating the valuable knowledge that the cultures of the humanists and scientists can bring to an understanding of the modern world. As we are all aware, the story of light is so all-embracing that this course included not only the science of light and optics, with lasers and photonics, but light in the open air, astronomy, religion, literature, art, architecture, film, stage, health and medicine, with several of the topics discussed by guest speakers. After a brief review of the story of light from its earliest beginnings, an outline of the course will be given along with the many contributions by the students and evaluation of their efforts.
\end{abstract}

Keywords: a course in understanding light past and present, properties of light and applications

\section{INTRODUCTION}

At this conference on Education in Optics and Photonics, I have elected to discuss the broad topic of the nature of light and the development of our general understanding of light. We all know that there is more to the story of light than simply its applications, although optics is vital in our everyday lives, and has had a pronounced effect on civilization, especially in the past few decades with the development of the laser and the field of photonics.

What is light? As Samuel Johnson (1709-84) put it so succinctly "We all know what light is, but it is difficult to tell what it is." And to quote Albert Einstein (1879-1955) about 1917 "For the rest of my life I will reflect on what light is!" We are all aware that we are creatures of light, life itself was born of light, and sight is our most important link to the world around us. Throughout the Ages, in the East and West, light meaning white, pure, life, love, wisdom, has played a vital role in religion and philosophy, in poetry and music, in art and science. Philosophers, poets, theologians, writers, artists, musicians, and scientists, all in their own way, have been trying to solve this 'Riddle of Light'.

Those of us in the study of light, optics, and photonics realize that we have a rich heritage of knowledge. I believe that light gives us a rare opportunity to bring to students the intellectual accomplishments of civilization with the hope of bringing together the two cultures of the humanists and scientists. It was forty years ago that the scientist and novelist Charles P. Snow, wrote of the arrogance of members of these two cultures creating a vast gulf in society, and that the lack of communication between the two groups could be fatal to the Western world. It seems to me that the story of light and its applications is a powerful example of consilience ${ }^{1}$ (the unity of knowledge) that may help to bridge this gap in today's world.

Before I discuss a seminar course that I have developed over these past three years for first year undergraduates, I would like to briefly review our understanding of light and optics and how this has come about.

\section{THE STORY OF LIGHT ${ }^{2}$}

\subsection{Divine Light and Early Understanding}

The story of light begins in remote antiquity. In western civilization it began with God, who said: "Let there be Light", and light appeared before the Sun or any other source of light. And light which provides visibility became the dominant symbol in ancient myths and religions, and finally the central theme of knowledge and understanding. Even in pre-Biblical times, light was the deity "Helios" the Sun, the source of life and abundance on Earth. In ancient Egypt the Sun-god Re was predominant and his divine son the Pharaoh was often shown being caressed by the Sun's rays which end in delicate hands. 
The early Greek philosophers were concerned with light and with the puzzle "How do we see?" Their ideas centered on the fire within the eye sending a beam of light rays travelling in straight lines to illuminate objects and the light then returns to the eye. With Plato (427-347 B.C.) the source of light is man's soul. He also introduced the idea of a light-carrying ether, a medium for the passage of light which fills all space. Rays of light were later adapted in many paintings of religious scenes, such as the Nativity, with rays from the heavens illuminating the scene, and of course the concept of rays remains with us today as a useful method for the design of lenses, mirrors, and more complex optics.

With the coming of the Dark Ages in western civilization, the center of knowledge moved to the East and Arabic world. There came the first suggestion by Alhazen (965-1041) that sight proceeds entirely by means of light entering the eye from illuminated objects around us. Leonardo da Vinci (1452-1519) agreed with Alhazen and expressed the notion that the eye far from being a source of light is a dark chamber into which an image of the world is projected. He re-introduced what is known as the 'camera obscura' of Alhazen, namely, a box having in the front wall a small pin-hole to permit light to enter, with the back wall serving as a screen for viewing the resulting image, which is inverted. The French philosopher René Descartes (1596-1650) agreed with these ideas about the eye and vision, and carried out experiments to show that the eye acts just like the camera obscura. He scraped the back side of a cow's eye to make it transparent, and observed the image produced as light passed through the pupil, iris, and lens to form an image on the retina. And lo and behold the image was inverted.

We may well ask "how is it that while the images on our retinas are inverted, we do not see people standing on their heads, that is upside down?" That story only unravelled in the 20th century, and the explanation relies on perceptual psychology and physiology, areas that are still being explored. Other fascinating effects include image retention, after-images, and contrast phenomena, as appear in op art.

\subsection{Cathedrals of Light}

Christian churches were built in the Romanesque style with small windows to provide some light and air. In the 12 th century, the use of exterior buttresses to support the walls of churches provided the possibility of incorporating large spans of glass in the structure. The Abbe Suger (1081-1151) took this opportunity to build the Abbey Church of St. Denis near Paris with walls of glass, bathing the interior in infinite shades of color with sunlight streaming through beautiful stained glass windows, depicting stories from the bible. In the following centuries, many churches and cathedrals were built in northern Europe and Great Britain in this Gothic style, full of stained glass windows as a method of bringing the Christian doctrine to the illiterate congregations.

This interaction of light and stained glass, conceived in medieval times, is unique in art and architecture, and depends on natural light, and the reflection, refraction, and absorption of light by the colored glass. Perhaps the most impressive stained glass is to be found in the rosary windows (the symbols of love) of the larger cathedrals such as Chartres, Reims, Toledo, and Notre Dame in Paris.

\subsection{Celestial Light}

From earliest times to the present day, people have been enchanted and mystified by the nightly splendor of the heavens, and have continued to ponder its hidden meaning. Different civilizations have embraced the celestial light in their mythologies and religions, and used the daily transit of the Sun to tell time, and the cycle of the Moon's phases to predict the seasons and form calendars. And the stars have served as a guide to travellers on land and sea for centuries.

For study of the positions and movements of Sun, Moon, stars and planets, simple observatories such as Stonehenge were built by various civilizations around the world. However, it was the development of the telescope and the microscope in the 17 th century that magnified our views of the heavens and of the infinitesimal world. Today the Hubble telescope in space brings galaxies from infinite distances to our screens, and the COBE satellite recently revealed the first light after the Big Bang creation of the Universe.

One of the earliest to turn a telescope to the heavens was Galileo Galilei (1564-1642). He saw spots on the Sun and noted that they changed their position from day to day, and thus determined that the Sun rotates on its axis. He saw craters on the Moon, rings around the planet Saturn, and moons circling Jupiter. From his observations Galileo confirmed Copernicus' findings that the Earth orbits the Sun, contrary to earlier beliefs. The Church banished his books, and the study of light found new homes in England, France and Holland.

In time, it was established that the blue of the sky was due to sunlight scattered by molecules in the atmosphere, with blue light being more efficienly scattered than yellow and red. The reds of dawn and sunset also arise from such scattering since we look through more atmosphere at the horizon, and the increased scattering of blue leaves mainly the red for us to see in the direction of the Sun. Rainbows are a daylight wonder of the heavens, and these are formed by the reflection of sunlight 
within the drops of rain together with the bending of light as it enters and exits the raindrops. In winter in the cold regions are seen brilliant 'sun dogs' on each side of the Sun, and often the complete halo around the Sun, all caused by ice crystals in the atmosphere.

The Aurora or Northern and Southern Lights are frequently seen in various forms, as moving rays, shimmering curtains, or broad bands of red and green light. These are caused by charged particles from the Sun being attracted to the north and south magnetic poles of the Earth and hitting the oxygen and nitrogen molecules in the atmosphere to make them glow. Major displays seem to occur every eleven years, when there is intense sunspot activity.

\subsection{Color is Light}

Isaac Newton (1642-1727) demonstrated that white light was composed of a spectrum of a myriad colors. With the coming of the plague in London when he was 23 years of age, Newton left London for his home in Woolthorp. While there, Newton not only developed his ideas on gravity, but he set up an experiment to show that sunlight (white light) can produce a rainbow of sorts, just as it does with raindrops. For his experiment, Newton let sunlight coming through a slit in a window fall on a prism of glass, and demonstrated that the white light is broken into many colors or a spectrum as seen on a screen. He deduced that white light consists of this combination of colors. Moreover, he showed that the colors in the spectrum are not divided any further, by putting parts of the spectrum through another prism, and the colors did not change.

Johann Wolfgang von Goethe (1749-1832), the German poet, dramatist, novelist and statesman, didn't believe Newton's results completely, and had different ideas about color. He believed that physiology played a role. Yet in Newton's experiment, all that happens is that sunlight passes through a glass prism to produce the spectrum of continuous changing color. Newton even went further and mixed simple colors to form other colors and even to form white. As an example, when the three colors of red, green, and blue are partially mixed (or superimposed), the red and green produce yellow, the blue and red make magenta, and green and blue lead to cyan. But when all three colors are mixed, the result is white. In fact, this combination of red, green, and blue colors is used today to provide the rich variety of colors seen on television screens: the screen is covered with tiny dots of red, green, and blue, packed closely together, and when the electrons hit these different spots, many colors are produced by mixing of the three colors.

\subsection{Literature, Music, and Art}

Writers were fascinated with light. With Alighieri Dante (1265-1321), love exists in that part of the soul formed by light; light is the source of all beauty, all intellectual activity, and it illuminates everything good. He stated: "Above the Creator is light and makes Him visible to every creature." Also in his poem The Divine Comedy the Inferno is dark and underground; when he begins to ascend Mount Purgatory it is dawn; and when he reaches Paradiso, it is infused with light continually.

In William Shakespeare's works (1564-1616), light means the Sun, life, passion innocence, truth, all that is strong and good. Its absence is foreboding and evil. In Othello, as the mistakenly jealous Othello is about to kill Desdemona in her sleep, he stands over her and ponders his imminent actions saying

\section{"Yet she must die else she'll betray more men, Put out the light and then put out the light."}

After killing her,

"Methinks it should be now a huge eclipse of sun and moon"

Anyone who has experienced the swift onset of complete darkness at the time of a total eclipse will appreciate the power of those lines.

Light was also extolled in music, as in The Magic Flute by Wolfgang Amadeus Mozart (1756-91). Before he died, the King of the Sun entrusted the powers of the shield of the Sun to Surastro the high priest of the Sun rather than to his wife the Queen of Night. A struggle between darkness and light continues throughout the opera, but the forces of light are eventually the victors and the triumphs of beauty and wisdom are praised. In Franz Joseph Haydn's (1732-1809) great oratorio, The Creation, there is a superb choral outburst in the C-major cord at the words "and there was light." This text is based in part on the blind John Milton's (1608-74) Paradise Lost with the memorable lines

\section{"Hail holy light, offspring of Heav'n first born, Or of th'Eternal Coeternal beam."}

It was at this time of the 17th century that mathematicians and natural philosophers or scientists began their serious interest in light. And with the advent of some understanding of light and its properties, writers ceased to believe in light as mystical, magical, and the substance of the soul. 
Artists have their own version of the aesthetics of light, and it is instructive to briefly examine how painters made use of light over the centuries. In the earliest paintings known, of about 15,000 BC in the Lascaux caves of southern France, a three-dimensional cast is given to the animals by the shadows on their underbellies, suggesting that the artists were aware of the effects of light and shadow. In the Faiyum paintings on the coffins of Egyptians, in vogue about the year $200 \mathrm{AD}$, the portraits are essentially of modern day technique, with light reflected in the eyes, shadows giving depth to the face, and jewelry sparkling with light. Then came the Dark Ages (about 500 to $1300 \mathrm{AD}$ ) with mainly two-dimensional, religious canvases, and it took almost a millenium before artists returned to painting in a truer life-like style. Finally, about 1300, Giotto resumed the use of shadows to bring depth to paintings, and proper geometry to indicate perspective. He was also aware of science, and used Halley's comet which appeared at that time instead of the star of Bethlehem in his Nativity scene. Caravaggio (1569-1609) was a master in the use of light and dark or chiaroscuro to portray depth and feeling. A magnificent example is his 'Supper at Emmaus' with the fingers and hands of Jesus in strong light and shadow. Early painters in Belgium such as Georges de La Tour (1593-1652) included the candle in paintings to illuminate their subjects.

The self-portraits of Rembrandt van Rijn (1606-69), made with thick layers of paint on a white base depict light from within himself. And Jan Vermeer's (1632-75) works with sunlight streaming through a window to highlight a simple scene express the thought that light radiates outward from its source to dispel darkness, - like the halos of the saints, and the stained glass windows of cathedrals of Europe. This thought was echoed by Dylan Thomas (1914-53) who referred to his poetry as "the record of my individual struggle from darkness towards some measure of light." One of $J$. M. W. Turner's (1775-1851) famous paintings 'The Snowstorm' is simply a scene of light and darkness, but it makes a tremendous effect. And the Impressionist Pierre-August Renoir (1841-1919), in 'A Woman on a Swing', plays with light and shade as light dapples through the trees to illuminate the scene. Claude Monet (1840-1926) produced several series of Reims Cathedral and of hay stacks, painted at different times of day and season. A decade later, rather than mixing paints on his palette, Georges Seurat (1859-91) used pointillism, a tight pattern of small dots of pure color, and let the viewer's eye do the mixing of colors. Vincent van Gogh (1853-90) lit up the sky in "The Starry Night". A painting by Giacomo Balla (1871-1958) the Italian Futurist, produced in 1909 when the streets of Rome were first illuminated with electrical light shows the power of electricity, not only with the brilliant colored chevrons but with the Moon subdued by the brighter artificial light. Finally, Rene Magritte (1898-1967) in his series 'Empire of Lights', portrayed the twenty-four hours of day, with a house in darkness, bedroom windows illuminated, a street lamp reflected in a pool of water, and the sky clearly in daylight. He felt strongly about light and dark and wrote: "I find this evocation of night and day is endowed with the power to surprise and enchant us all; I call this power poetry."

\subsection{Light and Health}

The lack of sunlight in polluted environments was known to cause rickets, a deformation of bone and cartilage due to deficiencies in vitamins during early growth, and it became necessary to supply children with Vitamin D from artificial sources (cod liver oil, milk) and artificial sunlight. In sunlight humans develop Vitamin D in their bodies by the action of light on chemicals in the skin. Tuberculosis, a germ disease causing severe coughing, loss of weight and sweating is also helped by rest in a dry and sunny climate. Seasonal affective disorder (SAD), or winter depression is more common in Nordic countries, and is treated with daily exposure to the full spectrum of light from fluorescent lamps. Too much sunlight is also a problem, causing sunburn mainly from ultraviolet light, and sunstroke from infrared or heat rays. Perhaps more serious today is skin cancer or cell damage resulting from overexposure to ultraviolet light, an increased danger when the ozone protective layer is thinned by pollutants.

\subsection{Some Characteristics of Light}

The reflection and refraction of light were studied by the early Greek philosophers. They knew that the angle of reflection is equal to the angle of incidence. Light falling on a transparent medium such as glass or water is partly reflected and some transmitted but noticeably bent in its path through the medium due to refraction. While the bending of a light beam as it passes from one medium to another was also known, it was Willebrord Snell (1591-1626) a Dutch scientist, and René Descartes who gave the formula for the angles of incidence and refraction. When an observer is in water looking up to the surface, the rays of light are bent, and as the angle of viewing becomes larger and larger to the normal, there finally is reached what is known as the critical angle, when the light is bent to coincide with the surface; at still larger angles the surface acts as a mirror of extremely high efficiency. This effect is called Total Internal Reflection, and has extremely important uses; when a light beam is incident at one end of a rod of glass or clear plastic, the light is transmitted without much loss to the other end even when there are many bends in the rod. Today, optical glass fibers, having the diameter of a human hair, carry our telephone conversations across continents and oceans by means of light transmitted through these fibers making use of total internal reflection. This major new development has come about from this simple concept of the critical angle for reflection of light travelling from a dense to a less dense medium. 
As a result of his various experiments in optics, Newton concluded that light is a stream of particles. Others, including the Englishmen Robert Hook and Thomas Young, Christiaan Huygens a Dutchman, and Augustin Fresnel a Frenchman, among others, refuted him and insisted that light is a wave. So began a controversy, "the Riddle of Light": Is light a wave or a particle? This puzzle lasted almost three centuries.

Many have played a role in finally bringing some understanding to this riddle. Foremost among them were Thomas Young (1773-1829) who discovered the principle of interference and Augustin Fresnel (1788-1827) who explained the diffraction of light. There are many observations demonstrating that light behaves as a wave. First of all, when a light beam is incident on an obstacle, we expect to see a sharp edge on a dark shadow where the object intercepts the beam. However, when the experiment is carried out, we see that the edge is not sharp, but a variation in intensity right at the edge, which can only be understood as caused by diffraction of the light wave. Thomas Young carried out an elegant experiment with light falling on two nearby parallel slits. When either slit is covered, so that light passes through only one slit onto a screen, an evenly illuminated screen is observed. However, when light from both slits is allowed to fall on the screen, we see instead a series of light and dark bands, known as interference fringes. It was this experimental result that convinced scientists beyond a doubt that light is a wave. In Young's experiment, although the waves start out in step from the two slits, they get out of step as they travel different distances to the various positions on the screen and interfere constructively and destructively. Similar interference patterns are readily demonstrated with water waves produced by dropping two stones in the water.

As we all know, Albert Einstein came into the picture later on in support of particles (photons or quanta). Some properties of light are best explained if light is assumed to be a wave, while others can only be understood if light has the characteristics of particles, and we conclude that light is a wave and a particle. This we call the Wave-Particle Duality. In a sense it seems to convey the impression that we really don't know what light is. Even Einstein remained in a quandry: "All the fifty years of conscious brooding have brought me no closer to the answer to the question 'What are light quanta?' Of course today every rascal thinks he knows the answer, but he is deluding himself."

The story of the wave nature of light so far has dealt primarily with research and findings in England, France, and Holland. But in Scotland, James Clerk Maxwell (1831-79) proved purely theoretically, while studying electricity and magnetism, that electric and magnetic fields propagate as waves and they travel at the speed of light. From this result he concluded that light is a disturbance in the form of (invisible) electric and magnetic waves travelling in step. Moreover the visible portion of light is only a small part of a whole spectrum of wavelengths from long radio waves, to infrared, visible, and ultraviolet waves, $x$-rays, and gamma rays, and so on. All of these waves of vastly different lengths and frequencies have specific properties, but all travel at the same speed, the speed of light, $3 \times 10^{8}$ meters per second.

\subsection{Relativity}

The acceptance of the wave theory of light demanded an equal acceptance of an all pervading ether, with all bodies, the Sun, Earth, and planets immersed in it. Thus, standing on the Earth which rushes along in its orbit around the Sun, and the Sun and planets travelling through space, we should experience an "ether wind" resulting in a Doppler shift in wavelength of a light source moving with the wind than one that is stationary. But when the Americans Albert A. Michelson (1852-1931) and Edward Morley (1838-1923) sent light in different directions, they always found the same speed of light. Much to their surprize and to everyone else repeating the experiment, the ether wind had no effect on the speed of light.

This dilemma culminated in 1905, with Albert Einstein asking "What would the world look like if I rode on a beam of light?" His answer was that for him, time would stand still, and space and all bodies embedded in it would contract. For Newton, the world looks the same to every observer, and in Einstein's view what you see and I see is relative to each of us, to our place and to our speed. We can communicate with one another at the speed of light, which is the same for all observers, and is the maximum speed, - a speed which material bodies cannot attain. And so was born the Theory of Relativity, with Space-Time replacing the ether. Its conclusions have profoundly changed our view of the Universe.

\subsection{Spectroscopy, quantum theory and the origin of light}

So far our story has skirted around the question of the origin of light, that is; how is light produced?. This was only revealed in the 20th century through spectroscopy and observations of the interaction of light and matter. One wonders why this study was left so late, since it is so fundamental.

It was known that the intensity and colour of light from a glowing hot body, whether coal or the Sun, was related to its temperature by a universal relation. At low temperatures, bodies appeared to be red, and at higher temperatures they became white. Our Sun with its surface at approximately $6,000^{\circ} \mathrm{C}$ has a maximum intensity at $540 \mathrm{~nm}$, in the yellow part of the spectrum. (It is noteworthy that our eyes are most sensitive to this region of the spectrum.) In 1900, the German physicist Max Planck (1858-1947) explained the relationship between intensity, wavelength and temperature by assuming that hot 
bodies do not emit their light energy continuously, but in finite amounts or quanta. This led Einstein to revive the particle theory of light in a new form, with energy quanta existing as real light-particles or photons, having energy equal to $h v$ (where $h$ is a constant and $v$ is a frequency), but no mass. And so the world entered the era of Quantum Theory.

It was also known that all elements emit light of specific colours or wavelengths that are characteristic of the element, - the so-called line spectrum of the element. This is essentially a fingerprint, since once the wavelength is known, the element is identified. This is how we know that the Sun and the distant stars are made of the same elements as known on Earth. But it was not understood why light was emitted in spectral lines.

The Danish physicist Neils Bohr (1885-1962) used the concepts of quantum theory with Ernest Rutherford's (1871-1937) model of the planetary atom to suggest that the electrons circling the positively charged nucleus were constrained to move in certain orbits (or stationary levels) in the space surrounding the nucleus. These orbits were at specific distances from the center, with the farthest out having the most energy. However, the electrons do not stay in these orbits or levels for long, but jump back to lower levels and finally to the lowest possible level nearest the nucleus. In jumping from the higher to the lower levels, the electrons give up their energy in the form of light. This is how all light is produced. No matter what the source, or whether a gas, liquid or solid, light is produced when an electron of higher energy makes a transition to a lower energy. With the study of this emission, considerable insight has been obtained into the structures of atoms, molecules, and all of matter, and their gaseous, liquid, and solid states. It is clear that spectroscopy, the study of spectra has been crucial in our understanding of light.

Another property of the process of light emission is the random nature of the emission, random in time and in direction. This process we call Spontaneous Emission. It was Albert Einstein who suggested that forced or Stimulated Emission is also possible. This occurs when an electron in an upper state is forced to make a transition to a lower state by having light incident on it. When this happens, the electron emits the light in the direction of the original incident light and in step with it, thus amplifying its intensity. So if we have a long tube of excited atoms, or enclose the system with mirrors at both ends so that the light bounces back and forth many times, light of high intensity, directionality and coherence is produced. This is the basic mechanism in the operation of Lasers.

Today, the study of light continues in laboratories and observatories around the world. While we understand much about the nature of light and its properties, we realize there is much more to the story I have just told; there is much to learn, and more to discover. And I believe that each discovery will not merely be an advance for Science, but a contribution to Civilization. So we continue to ask: "What is light and what can we do with it?"

\section{SEMINAR PROGRAM - 'THE RIDDLE OF LIGHT'}

From this brief review of the story of light, we see that it is rich in mythology, religion, the arts, and the natural and life sciences. And we can appreciate the statement of Kenneth Clark who said: "All of the greatest exponents of civilization have been obsessed by light."

Thus, when a new course for first year students entering the Arts and Sciences Program was inaugurated at the University of Toronto five years ago, I decided to take up this challenge and developed a seminar course for the study of light. First a few words about the overall new program. About 80 courses (two hours per week, year-long ) on a variety of topics were offered as electives to students enrolled in either the liberal arts or science disciplines. Only 20 students were permitted to participate in any one course, and the planning of each course was the responsibility of each professor.

I have developed and taught a course titled "The Riddle of Light". From the above very brief account of our past and present understanding of light, it is obvious that there is a wealth of material for study. In the three years that I have taught this course, the students were from various disciplines and each class was attended by an equal number of men and women.

\subsection{Topics selected for discussion}

\section{Light and civilization}

early history and ideas on vision

light in religion

light in literature

light in art

quantum theory and the source of light

theory of relativity 
Light: Its properties, optics and photonics a review of waves and superposition of waves transverse (light) and longitudinal (sound) waves electromagnetic waves and electromagnetic spectrum diffraction, interference, and resolution polarized light speed of light, $c=\lambda v$ ( $\lambda$ is wavelength and $v$ is frequency) spontaneous and stimulated emission: lasers optical instruments (lenses, mirrors, gratings, microscopes, telescopes) fiber optics, electro-optics, nonlinear optics

\section{Light in nature and applications of light}

light in the open air (sky, clouds, rainbows, mirages, effects of ice crystals) the heavens, astronomy, astrophysics, cosmology light in architecture film, the medium of light light in health and medicine lasers and use in science, communications and medicine

\subsection{Seminar schedule}

\section{First Term}

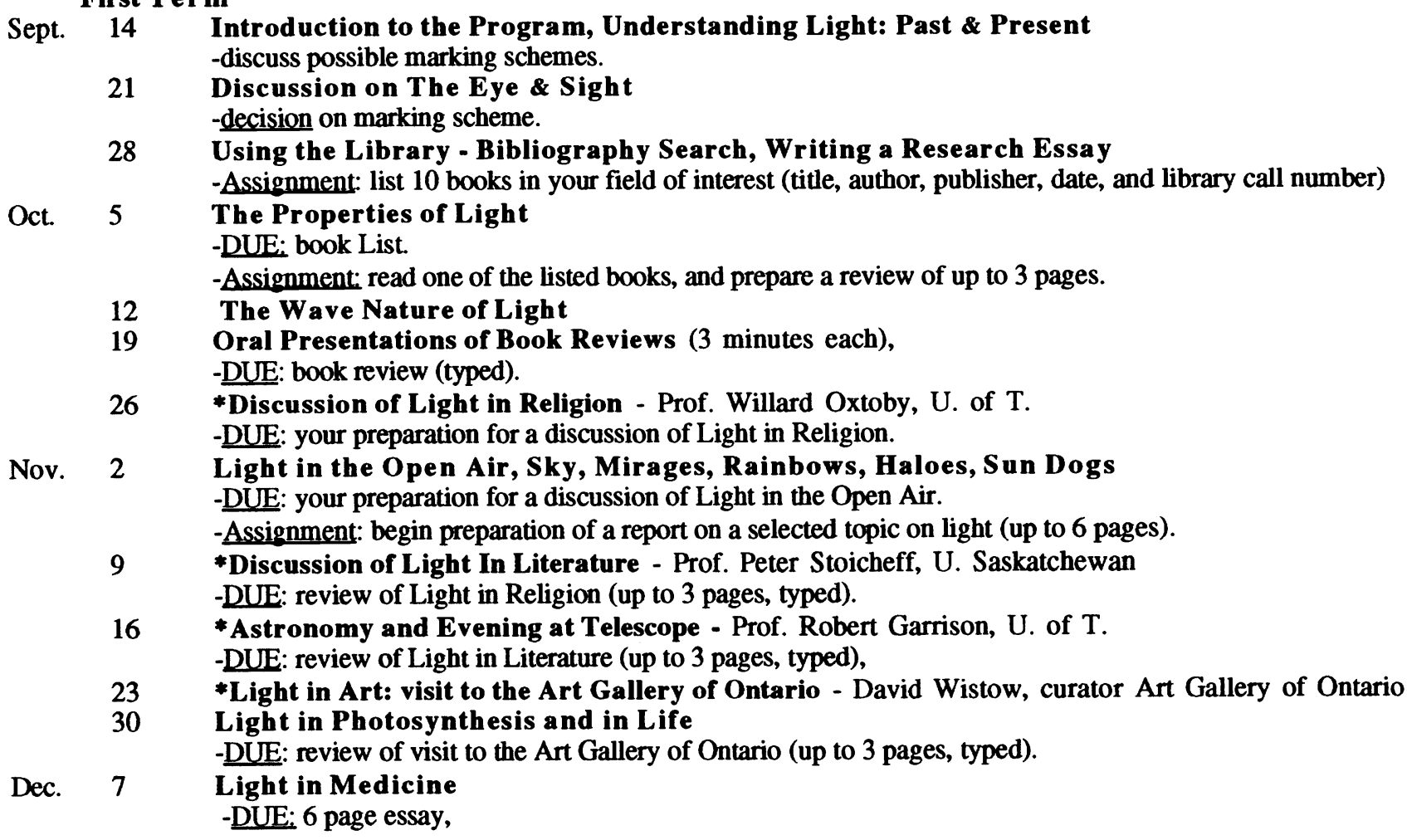

\section{Second Term}

Main Assignment for Second Term; select a new topic on light, and prepare an oral presentation of $20 \mathrm{~min}$. followed by a $10 \mathrm{~min}$ discussion. Finally, prepare a 10-page essay on this topic. (Oral presentations to be given Feb. 22 to Apr. 4; essays due Apr. 11.)

Jan. 11 Origin of Light, Spectroscopy, and Quantum Theory -Assignment: what was new and what was surprising to you in the Quantum Theory?

18 *Lasers, Applications and the Information Age (Visit to Labs) -Prof. Henry van Driel, U. of T -DUE: queries on Quantum Theory.

-Assignment: prepare a report for discussion of Light in Film \& Theater. 
25 *Discussion of Light in Film and Theater - Profs. Keil \& Freund, U. of T. -DUE: your preparation for a discussion of Light in Film \& Theater.

Feb. 1 Light and the Theory of Special Relativity -Assignment: review topics in Quantum Theory and Relativity for discussion.

8 *Open Discussion of Light, Quantum Theory and Relativity

-Assignment: prepare a 3-page report for discussion on Light in Architecture

15 *Discussion of Light in Architecture - Prof. Brigitte Shim, U. of T. -DUE: your preparation for Light in Architecture

22 Begin Oral Presentations and Discussions (3 each week), -Assionment: prepare a review of one of the presentations (up to 3 pages, typed).

29 Oral Presentations and Discussions, -DUE: review of one of the previous presentations, -Assignment: prepare a review of one of the presentations (up to 3 pages, typed).

Mar. 7 Oral Presentations and Discussions,

-DUE: review of one of the previous presentations,

14 Oral Presentations and Discussions, -DUE: review of one of the previous presentations,

21 Oral presentations and discussions -DUE: review of one of the previous presentations, 28 Oral Presentations and Discussions, -DUE: review of one of the previous presentations,

Apr. 4 Oral Presentations and Discussions, -DUE: review of one of the previous presentations, 11 -DUE: 10 page essay

3.3. Marking scheme

Fall Winter

Class Participation:

$\begin{array}{lll}\text { attendance, questions, discussion } & 5 \% & 10 \%\end{array}$

Class Assignments:

book list, book review (oral \& written), brief reports $\quad 15 \%$ and reviews of student presentations

Essay / Report a selected topic on light, (6 pages)

Oral Presentation

a second (and substantially different) selected topic on light, $20 \mathrm{~min}$. presentation, $10 \mathrm{~min}$. discussion.

Essay on the Above Oral Topic: 10 pages including additions from class discussions.

$15 \%$

$15 \%$

Total $\quad \underline{35 \%} \quad \underline{65 \%}$

\section{ORAL PRESENTATIONS}

- Topic to stress light whatever the subject matter or application; each to last up to 20 minutes, during which time the blackboard, the overhead and slide projector, or handouts may be used.

- Marking will be based on content, knowledge of the topic, efficiency in use of the allotted time, and presentation.

- A discussion period of up to 10 minutes will follow for comments and questions. Participation of the group will be assessed, as will the effectiveness of the speaker in encouraging discussion, and in dealing with questions.

\subsection{Marking scheme for oral presentations}

\section{Content:}

Introduction Relation to the course on light? 3

Main Body

Conclusion/Summary

Have you informed/educated us?

Stress the importance of light. 
Presentation:

Confidence/Enthusiasm

Time:

Use of slides, models, etc.

Has your presentation inspired us?

2

3

Use of the 20 minutes

2

Discussion:

Handling of questions

and comments.

Did you lead the discussion?

Did you know your topic?

Use of the $10 \mathrm{~min}$.
Total
$30 / 2=15 \%$

\section{WRITING ASSIGNMENT: ESSAY ON LIGHT}

Topic: $\quad$ subject matter must be the same topic as the oral presentation, with emphasis on Light.

- additional material may be included, based on ideas from the discussion, or from further study you may have carried out on this topic.

Length: $\quad$ up to 10 pages, typed and double-spaced.

- figures may be added, either at the end or throughout the text (but are not included as part of the 10 pages).

Format: - any style will be acceptable, that is, you may use various headings, or use the essay form if you prefer,

- each page must have approx. $2.5 \mathrm{~cm}$ margins, top, bottom, and sides, and must be numbered.

- a bibliography is required, and direct quotations must be referenced.

5.1. Marking scheme for theme paper

Content:

Introduction

Main Body: Facts, explanations, with emphasis on light, including different points of view, as well as author's view.

Conclusion/Summary

12

2

Composition:

Progressive and specific thought throughout, with little repetition; good use of words

Enhancement of Presentation:

That is, any additional material, or more details than given in the oral presentation

Bibliography:

Is it sufficient? Are good quotes included?

Penalty for Lateness: Deduct $2 /$ day

Total

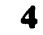

$25 \%$

\section{STUDENT ORAL PRESENTATIONS and ESSAY TOPICS}

\section{Light in nature}

Play in the heavens: Light reflection and refraction creating natural wonders

Starlight

Light in the open air

The mysterious northern lights

Solar energy

Phosphorescence

Optical illusions

Light in the development of life

The speed of light

Light and civilization

Light in the old religions

Light in the Aztec culture

Light and healing in various cultures 
The essence of light in Greek mythology

Sun Gods of the Inca civilization and their use of light

Light in ancient Egyptian religion

The integral role of the sun in native American cultures

Light and optics in the middle ages

Robert Grosseteste and optics

Two great legacies: Newton and Einstein

Nicholas Copernicus

The candle and the advent of indoor lighting

Physiological effects of light

\section{Light in art and architecture}

Stained glass

Light in cinematography

Light in film noir

The medium and the message: Light symbolism in motion pictures

The light of the impressionists

Michelangelo da Carravagio and chiaroscuro

Louis Kahn: An architect of light

Op art

Photography

\section{Applications of light}

The magic of stage lighting

The telescope

Light in our homes

A brief history of telecommunications

Ultraviolet light and its effects

The basic physics and chemistry of modern black and white photography

Light and health

\section{SUMMARY}

7.1. Objective

To study the nature of light and its properties with applications of optics and photonics, and their effects on civilization and the modern world.

7.2. Method

- learned how to search for bibliography in the library,

- had a discussion on writing a research essay,

- BPS gave numerous seminars and handed out notes,

- experts in various fields of light were invited to present seminars

- visited the Art Gallery of Ontario, with tour by a curator,

- each member gave a review ( $3 \mathrm{~min}$.) of a selected book on light,

- each member submitted a 6 page paper on a chosen topic of light

- each member gave presentations (20 min.) and led discussions (10 min.) on a chosen topic of light,

- each member submitted brief reviews of the weekly oral presentations of colleagues,

- each member submitted a 10 page paper on the topic of the oral presentation.

\section{ACKNOWLEDGEMENTS}

The support of the University of Toronto and of Photonics Research Ontario (PRO) is gratefully acknowledged.

\section{REFERENCES}

1. E. O. Wilson, Consilience,Vintage Books, New York, 1998.

2. R. Greenler, Rainbows, Haloes, and Glories, Cambridge University Press, Cambridge, 1980

D. Park, Fire in the Eye, Princeton University Press, Princeton, 1997.

S. J. Williamson and H. Z. Cummins, Light and Color in Nature and Art, John Wiley and Sons, New York, 1983.

E. Wolf, "Einstein's Researches on the Nature of Light," Optics News 5, 24-39, 1979. 